\title{
FUNCTIONAL OUTCOMES AMONG PRETERM INFANTS WITH INTRAVENTRICULAR HEMORRHAGE
}

\author{
V. Bozzetti ${ }^{1}$, S. Malguzzi ${ }^{1}$, G. Paterlini ${ }^{1}$, C. Colonna ${ }^{2}$, G. Kullmann ${ }^{2}$, P.E. Tagliabue ${ }^{1}$ \\ ${ }^{I}$ NICU, ${ }^{2}$ Infantile Neuropsychiatry, San Gerardo Hospital, Monza, Italy
}

This work evaluated the neurodevelopmental and functional outcomes of premature infants with intraventricular hemorrhage (IVH). These outcomes were analyzed for their association with IVH severity.

Methods and materials: In the years 2008 and 2009 we performed cerebral ultrasounds of all children with gestational age less than 32 weeks and/or birthweight under 1500 grams. We evaluated the motor outcome (infant cerebral palsy versus normal status) and the neurodevelopmental outcome (Griffith score at $12^{\text {th }}$ month of corrected age) of all children with IVH (classificated according to Papile).

Results: In 2008 and 2009 the newborn evaluated were 108 and 90 respectively (both inborn and outborn).

\begin{tabular}{|l|c|c|}
\hline \multicolumn{1}{|c|}{} & 2008 & 2009 \\
\hline Prevalence of IVH (\%) & 28,9 & 20,4 \\
\hline Prevalence of IVH $>$ 2nd grade (\%) & 12,2 & 6,5 \\
\hline Prevalence of PCI (\%) & 27,3 & 33,3 \\
\hline Total (n) & 108 & 90 \\
\hline [IVH and PCI PREVALENCE]
\end{tabular}

The correlation between severity of IVH and occurence of PCI was of no statistical significance $(p<0,14)$.

The mean IQ was lower in infants with IVH of first grade than in infants with IVH of second grade or more (101 and 67 respectively). This difference occurred also in all the single items of the Griffith score (106-89102-102-108 and 61-54-72-68-82 respectively).

Conclusions: Even though the difference is not of statistical significance,the PCI is more frequent in infants with severe IVH.

The mean IQ correlates with the severity of the IVH.

Preventing cerebral hemorrhage may reduce negative long-term outcomes. 